\title{
THE THEORY OF TRANSFINITE RECURSION ${ }^{1}$
}

\author{
BY M. MACHOVER
}

Communicated by P. R. Halmos, April 24, 1961

During recent years there has been growing interest in logical calculi with infinitely long expressions (see e.g. $[2 ; 4 ; 5]$ ). However, all results obtained so far, many of them very remarkable, are semantic in character. The reason is that, while the syntax of the "classical" calculi could be "Gödelized" and then studied with the aid of the theory of recursive functions, no such procedure has so far been devised for the infinitistic calculi. To do this, one should possess transfinite analogues of the theory of recursive functions and of arithmetization.

In the investigations reported here, such analogues are constructed and applied to infinitistic calculi.

Let $\omega_{\alpha}$ be an arbitrary, but fixed, regular initial ordinal. By ordinals we shall mean ordinals $<\omega_{\alpha}$, by a sequence - a well ordered sequence similar to an ordinal, by the length of a sequence-its order type, and by a function - a function whose (single) argument ranges over the set of all sequences (of a fixed length) of ordinals and whose values are ordinals.

$\omega_{\alpha}$-recursiveness. Our first aim is to explicate the notion $a$ function whose value, for each given value of its argument, is "calculable" in a sequence of steps. This is done with the aid of a formalism (with infinitistic rules of formation and transformation) which is analogous to Kleene's formalism for recursive functions. (See [3, Chapter XI].) We use much of the metamathematical terminology of [3], in a sense analogous to that in which it is used there.)

Primitive symbols. " =", "'” (stroke), "sup" (the supremum operator), "0", a variable " $x_{\xi}$ " for each $\xi<\omega_{\alpha}$, a function letter (f.l.) " $f_{\xi}$ " for each $\xi<\omega_{\alpha}$.

Numerals. 0 followed by a sequence of length $\beta$ of strokes is the numeral for $\beta$. We denote it by $\beta$.

Terms. (a) Each numeral is an (atomic) term.

1 This is a preliminary report of results obtained in a doctoral dissertation prepared at the Hebrew University under the supervision of Dr. M. O. Rabin. The author wishes to take this opportunity to thank Professor A. Mostowski for valuable suggestions made during a period spent by the author at Warsaw University as a UNESCO Fellow. 
(b) A variable followed by a sequence of strokes is an (atomic) term.

(c) If $\left\{r_{\xi}\right\}_{\xi<\beta}$ is a sequence of terms and $f$ is a f.l. then $f\left(r_{0}, r_{1}, \cdots, r_{\xi}, \cdots\right)$ is a term.

(d) If $r$ is a term, $x$ is a variable and $y$ is a numeral or a variable, then $\sup _{x<y}(r)$ is a term.

(e) No others.

In our formalism (in contrast to Kleene's) there are bound occurrences of variables; each occurrence of $x$ in $\sup _{x<y}(r)$ is bound, except its occurrence as the $y$ under the sup, if $y$ happens to be the same as $x$.

A numerical equation is an equation of the form

$$
f\left(z_{0}, z_{1}, \cdots\right)=z \text {, }
$$

where $f$ is a f.l. and $z, z_{0}, z_{1}, \cdots$ are numerals.

An ascent is a sequence of numerical equations of the form

$$
\begin{aligned}
& f\left(0, z_{0}, z_{1}, \cdots\right)=z^{(0)}, \quad f\left(1, z_{0}, z_{1}, \cdots\right)=z^{(1)}, \cdots, \\
& f\left(\xi, z_{0}, z_{1}, \cdots\right)=z^{(\xi)}, \cdots .
\end{aligned}
$$

The supremum of the ascent (1) is the numeral for the least ordinal which is not smaller than any ordinal whose numeral is a right hand side of a member of the ascent (1). The term $\sup _{x<y} f\left(x, z_{0}, z_{1}, \cdots\right)$ where $x$ is any variable and $y$ is the numeral for the length of (1), is said to correspond to (1).

We have three rules of deduction (but no axioms):

$\left(\mathrm{R}_{1}\right)$ To pass from an equation $e$ to an equation $d$ resulting from $e$ by a simultaneous substitution of numerals for free occurrences of variables.

$\left(\mathrm{R}_{2}\right)$ To pass from an equation $r=s$ without free variables (the major premise) and a sequence of numerical equations (the minor premises) to an equation $r=s_{1}$, where $s_{1}$ is obtained from $s$ by simultaneously replacing in $s$ each part, which is the same as the left hand side of one of the minor premises, by the corresponding right hand side.

$\left(\mathrm{R}_{3}\right)$ To pass from an equation $r=s$ (the major premise) and a sequence $T$ of ascents (the members of the members of $T$ being the minor premises) to an equation $r=s_{1}$, where $s_{1}$ arises from $s$ by simultaneously replacing each term in $s$ which corresponds to one of the ascents of $T$ by the supremum of that ascent.

(The $s_{1}$ in $\left(R_{2}\right)$ and $\left(R_{3}\right)$ are not uniquely determined, if some of the minor premises are "contradictory".) 
We can now define deduction (in tree form) in analogy with [3]. A deduction may have infinitely many branches (however, less than $\boldsymbol{\aleph}_{\alpha}$ ) but each branch is finite in length. Also, in analogy with [3], we can define the notion the function $\phi$ is $\omega_{\alpha}$-recursive in the sequence of functions $\psi_{0}, \psi_{1}, \cdots, \psi_{\xi}, \cdots$.

$\omega_{\alpha}$-recursiveness is transitive in the sense of

THEOREM 1. If $\left\{\phi_{\nu}\right\}_{\nu \leqq \kappa}$ is a sequence of functions such that $\phi_{k}$ is $\phi$ and, for each $\nu \leqq \kappa$, either $\phi_{\nu}$ is one of the functions $\psi_{\mu},\left(\mu<\lambda<\omega_{\alpha}\right)$, or $\phi_{\nu}$ is $\omega_{\alpha}$-recursive in $\left\{\phi_{\mu_{\nu_{\tau}}}\right\}_{\tau<\rho_{\nu}},\left(\mu_{\nu_{\tau}}<\nu\right.$ for $\left.\tau<\rho_{\nu}\right)$, then $\phi$ is $\omega_{\alpha}$-recursive in $\left\{\psi_{\mu}\right\}_{\mu<\lambda}$. (Cf. [3, p. 270].)

If $\phi$ is $\omega_{\alpha}$-recursive in an empty sequence of functions, we say that $\phi$ is an $\omega_{\alpha}$-recursive function.

It can be shown that all functions commonly used in ordinal arithmetic are $\omega_{\alpha}$-recursive, if their arguments be restricted to ordinals (in our sense).

A predicate $S\left(x_{0}, x_{1}, \cdots\right)$ over ordinals is $\omega_{\alpha}$-arithmetical if it is equivalent to a predicate of the form

$$
Q_{1} \cdots Q_{n} R\left(y_{1}, \cdots, y_{n}, x_{0}, x_{1}, \cdots\right),
$$

where $n$ is finite, $Q_{k},(k \leqq n)$, is either $\left(y_{k}\right)$ or $\left(E y_{k}\right)$ and $R$ is an $\omega_{\alpha}$ recursive predicate, defined over ordinals.

$\omega_{0}$-recursiveness is the ordinary "classical" notion of recursiveness.

Arithmetization of transinite formalisms. From now on let $\alpha=\gamma+1$ be of the first kind and let the axiom of constructibility be assumed.

For the purpose of arithmetization, we must be able to assign to each sequence of ordinals a single ordinal (a Gödel number) in a one-to-one way, so that the predicate $\mathrm{Sq}(x)$ (meaning: $x$ is a Gödel number of some sequence) and the functions $L(x)$ and $M(x, y)$ (which, when $\mathrm{Sq}(x)$ holds, give the length and the $y$ th member, respectively, of the sequence whose Gödel number is $x$ ) be $\omega_{\alpha}$-recursive. This can, indeed, be done.

Consider the case $\alpha=1$.

Let $\left\{r_{\xi}\right\}_{\xi<\omega_{1}}$ be the set of all real numbers, well ordered according to their order of generation by Gödel's $F$-function (for the definition of $F$ see [1]).

For each $\beta<\omega_{1}$ there exist well orderings of type $\beta$ of all natural numbers $n<\beta$. Among them let $W_{\beta}=\left\{n_{\beta, \xi}\right\}_{\xi<\beta}$ be first in order of generation.

Given a sequence $Z=\left\{\zeta_{\xi}\right\}_{\xi<\beta}$ of ordinals, we let correspond to it the sequence $R=\left\{r_{\xi_{\xi}}\right\}_{\xi<\beta}$ of reals. Let $S=\left\{s_{k}\right\}_{k<\min (\omega, \beta}$, be the sequence 
of reals such that $s_{n_{\beta, \xi}}=r_{\zeta_{\xi}}$ for all $\xi<\beta$. To $S$ we let correspond a single real $s=r_{\eta}$. (This last correspondence is the one used in the well known proof of the equality $c^{\aleph_{0}}=c$.) To the ordered pair $\langle\eta, \beta\rangle$ we let correspond the ordinal $\tau=P^{\prime}\langle\eta, \beta\rangle$. (For the definition of $P$ see [1].)

Now, $\Phi(z)=\tau$ is a one-one correspondence between the set of all sequences of ordinals and a certain set of ordinals. We call $\tau$ the Gödel number of $Z$.

A similar procedure is possible for any $\alpha$ of the first kind. The following theorem can be proved:

THEOREM 2. The predicate $\mathrm{Sq}(x)$ and the functions $L(x)$ and $M(x, y)$ are $\omega_{\alpha}$-recursive, and their recursive definitions can actually be given explicitly, provided we use single symbols for $\omega$ and $\omega_{\alpha-1}=\omega_{\gamma}$.

We can now arithmetize our formalism and obtain analogues of the basic theorems of the theory of recursive functions (the enumeration and normal form theorems). However, no proper analogue seems to exist of the notion of primitive recursivity.

A typical application to infinitistic calculi is:

THEOREM 3. Let $\alpha$ be the first kind and let $F_{\omega_{\alpha}, \omega_{\alpha}}$ be an infinitistic first order language (having a supply of predicate letters and $\boldsymbol{\aleph}_{\alpha}$ individual variables. Negation, and conjunctions of sequences of wffs are allowed, as well as general quantification over sequences of free individual variables). Then the class of all satisfiable formulae of $F_{\omega_{\alpha^{\prime}} \omega_{\alpha}}$ is not $\omega_{\alpha^{-}}$ arithmetical.

This implies a negative solution of a problem raised by Henkin (in the Warsaw Symposium, 1959) concerning the completeness of a set of axioms for $F_{\omega_{1}, \omega_{1}}$. However, our solution depends in an essential way on the axiom of constructibility.

\section{REFERENCES}

1. K. Gödel, The consistency of the axiom of choice and of the generalized continuum hypothesis with the axioms of set theory, Princeton, N. J., Princeton University Press, 1940.

2. C. R. Karp, Formalizations of functional languages with wffs of infinite length, Abstract 542-57, Notices Amer. Math. Soc. vol. 5 (1958) pp. 172-173. 1952.

3. S. C. Kleene, Introduction to metamathematics, New York, D. Van Nostrand,

4. A. Tarski and D. Scott, The sentential calculus with infinitely long expressions, Colloq. Math. vol. 6 (1958) pp. 165-170.

5. A. Tarski, Remarks on predicate logic with infinitely long expressions, ibidem, pp. 171-176.

The Hebrew University of Jerusalem, Israel 